\title{
COVID-19 pandemic and psychological wellbeing among health care workers and general population: A systematic-review and meta-analysis of the current evidence from India
}

\author{
Rajesh Kumar Singh $^{\text {a }}$, Ram Bajpai ${ }^{\text {b }}$, Pradeep Kaswan ${ }^{\text {a, * }}$ \\ ${ }^{\text {a }}$ P.D.U. Medical College, Churu-331001, Rajasthan, India \\ ${ }^{\mathrm{b}}$ School of Medicine, Keele University, Staffordshire, UK
}

\section{A R T I C L E I N F O}

\section{Keywords:}

COVID-19

Mental health problem

India

Systematic review

Meta-analysis

\begin{abstract}
A B S T R A C T
Introduction: Coronavirus disease 2019 (COVID-19) was declared as pandemic and measures adopted for its control included quarantine of at-risk, isolation of infected along with other measures such as lockdown, restrictions on movement, and social interactions. Both the pandemic and these measures have the potential to cause mental health problems among individuals.

Objective: The present study aimed to investigate and estimate the prevalence of psychological well-being, particularly from an Indian perspective using systematic review and meta-analysis of existing literature. Methods: We searched in the PubMed database, starting from the onset of the current pandemic and until 10th October 2020 to synthesize evidence on mental health outcomes from India. DerSimonian and Laird method of the random-effects meta-analysis was employed and heterogeneity between studies was assessed using the Chisquare based Cochran's Q statistic and I-squared $\left(\mathrm{I}^{2}\right)$ statistics.

Results: The pooled prevalence of stress in nine studies was 60.7\% (95\% CI: 42.3\%-77.7\%), depression in eight studies was $32.7 \%$ (95\% CI: 24.6\%-41.3\%), anxiety in six studies was 34.1\% (95\% CI: 26.3\%-42.3\%) and sleep disturbances in six studies was $26.7 \%$ (95\% CI: $13.9 \%-41.8 \%$ ). As expected, high heterogeneity was observed in the above-mentioned outcomes. Sub-group analysis showed that Health Care Workers (HCWs) had a higher prevalence of stress, anxiety, depression \& psychological distress in comparison to the general population. Conclusion: A significant impact on psychological well-being during COVID-19 was observed in India as common adverse outcomes were stress (61\%), psychological distress (43\%), anxiety (34\%), depression (33\%), and sleep disturbances (27\%). Thus the COVID-19 pandemic represents an unprecedented threat to mental health, which should become a priority for public health strategies.
\end{abstract}

\section{Introduction}

The World Health Organisation (WHO) declared COVID-19 outbreak caused by Severe acute respiratory syndrome-coronavirus-2 (SARS-CoV2) as a Public Health Emergency of International Concern (PHEIC) on $30^{\text {th }}$ January 2020 and was officially labeled a pandemic on $11^{\text {th }}$ March $2020 .^{1,2}$ Due to the disease being transmitted through close contact between humans, extreme social distancing measures have been used to prevent its further spread. Lengthy lockdowns have been imposed in many countries to reduce the exponential spread of the virus and to alleviate pressures on healthcare systems. The government of India (GOI) imposed a complete lockdown with only essential services being functional from March 23 midnight. Most of the companies have encouraged their employees to "work from home"; however, no such provisions were offered to healthcare workers (HCWs). ${ }^{3}$

Both the pandemic and the lockdown measures have the potential to cause considerable panic and stress. The current COVID-19 pandemic itself is likely to evoke fear of infection, concerns regarding disease and death, and anxiety/stress about future health and economic uncertainties. ${ }^{4}$ This panic and stress are further raised by conflicting information about the coronavirus, the absence of a medical cure and vaccine, electronic and print media buzz regarding the COVID-19 deaths, infection rates, overcrowded hospitals, and other negative information about the pandemic. ${ }^{5}$

\footnotetext{
* Corresponding author.

E-mail address: pradeepkaswan3059@gmail.com (P. Kaswan).
} 
Along with the uncertainty and unpredictability of the current pandemic, lockdown and physical-distancing might lead to social isolation, loss of income, loneliness, inactivity, limited access to basic services, increased access to food, alcohol, and online gambling, and decreased family and social support, especially in older and vulnerable people. Racial and ethnic disparities in the incidence and associated mortality of COVID-19 have been exacerbated. ${ }^{6}$ The downturn in the economy caused by COVID-19 will lead to unemployment, financial insecurity, and poverty, which hinder access to health services (especially in countries with insurance-based systems), thereby having deleterious effects on physical and mental health and quality of life of its individuals. $^{7}$ The economic breakdown that is likely to occur in the aftermath of the pandemic could exacerbate health-care disparities and will probably disproportionately affect socially disadvantaged patients, including those from ethnic minorities. ${ }^{8}$

Mental health concerns and treatment usually take a backseat in settings with constrained resources that were geared for pandemic containment. History suggests that any infectious disease outbreak or pandemic brings with it a major setback in the mental health front. ${ }^{9}$ Sooner or later, health systems will be faced with widespread demand to address these COVID-19-related mental health needs. International organizations, including WHO, advocate for the integration of mental health and psychosocial support into the COVID-19 response. ${ }^{10} \mathrm{UN}$ policy brief suggests that investments now will reduce the mental health effects later. ${ }^{11}$

Current COVID-19 Pandemic has reached a level of a humanitarian crisis with over 50 million confirmed cases and 1,261,075 deaths reported globally to date while in India over 8.5 million confirmed cases and 127,059 deaths reported (Up to $10^{\text {th }}$ November 2020). ${ }^{12}$ During this period multiple studies have been conducted to assess the mental health status of various populations in different regions of India but there is limited evidence on the overall burden of mental health problems. This is important to address the needs and gaps in mental health care in India.

With the above-mentioned background, we planned to systematically review existing literature reporting the prevalence of psychological wellbeing (such as stress, anxiety, depression, psychological distress \& sleep disorders, etc.), particularly from the Indian perspective as a limited evidence synthesis published so far.

\section{Material and methods}

The present systematic literature review was performed following the guideline of the recommendations of the Preferred Reporting Items for Systematic Review and Meta-Analysis (PRISMA) guidelines. ${ }^{13}$

\subsection{Data source \& search strategy-}

Studies were identified by searching Medline (via PubMed) database. Only papers published in English \& from India were included. The period of paper searching was till $10^{\text {th }}$ October 2020 starting from the onset of the current pandemic ( $31^{\text {st }}$ December 2019). The search was performed on $10^{\text {th }}$ October 2020.

The search terms that were used were: ("mental health disorders"[Title/Abstract] OR "mental health"[Title/Abstract] OR "mental illness"[Title/Abstract] OR "mental health problem"[Title/Abstract] OR "psychiatry"[Title/Abstract] OR "stress"[Title/Abstract] OR "anxiety"[Title/Abstract] OR "depression"[Title/Abstract] OR "sleep disorder"[Title/Abstract]) AND ("COVID-19"[Title/Abstract] OR "SARSCoV-2"[Title/Abstract]) AND ("India"[MeSH Terms] OR "India"[All Fields] OR "India's"[All Fields]).

\subsection{Inclusion and exclusion criteria-}

We included research studies conducted among the general population or healthcare workers based on the following inclusion and exclusion criteria.
The criteria for studies to be included in the systematic review: (1) studies mentioning the prevalence of stress, anxiety, depression, sleep disturbance among the Indians during the COVID-19 pandemic in health care professionals and general population, (2) observational study design (such as cross-sectional, case-control, and cohort studies), (3) studies available with full text and (4) written in English language only as we consider that almost all the medical research studies conducted in India are published in English. The criteria for excluding a study were: (1) review articles, (2) case reports, (3) case series, (4) editorials or letters, (5) studies with population from outside India, and (6) articles with the un-availability of the full text.

\subsection{Study selection-}

One investigator (PK) screened titles and abstracts to exclude irrelevant articles and further examined the remaining full-text articles to determine compliance with inclusion criteria, and further screened relevant articles for additional reviews and examined full-text articles. The senior author (RS) double-checked all the included articles, and any dispute was resolved by consensus of both.

\subsection{Quality assessment-}

Two reviewers (PK \& RB) individually assessed the quality of data of the included studies using the National Institute of Health (NIH) quality assessment tools ${ }^{14}$ and any dispute was resolved by consensus. The NIH quality assessment tools were designed to assist reviewers in focusing on key concepts for critical appraisal of the internal validity of an article. Quality reviewers could select "yes," "no," or "cannot determine (CD)/not reported (NR)/not applicable (NA)" in response to each of 14 items, mentioned in the tool. The overall quality of included studies was rated as good, fair, and poor. Further, we incorporated these studies in the analysis and synthesis of results.

\subsection{Data extraction-}

Data from all included studies were collected into a standardized data extraction table (in Excel sheet) by the first reviewer (PK) and second reviewer (RB) independently checked for accuracy of extracted data. The third reviewer (RS) was consulted in case of any dispute between reviewers. The following variables were collected: 1) first author and period of publication, 2) state or area of the country, 3) study design, 4) total participants and target population 5) mean or median age and age group range, 6) the number of male participants and their $\%, 7)$ sampling or data collection method, 8) assessment tool and their cut-off, and 9) prevalence of symptoms of stress/depression/anxiety/ psychological distress/sleep disorders.

\subsection{Data synthesis-}

Prevalence was calculated for stress, anxiety, depression, and other outcomes including health care workers and general population subgroups. Prevalence was calculated as the ratio between the total numbers of reported cases over the total study population and presented as the number of cases per 100 population. Random-effects meta-analysis, using the DerSimonian and Laird method was employed on individual study estimates to obtain a crude summary estimate for prevalence. ${ }^{15}$ Standard errors and $95 \%$ confidence intervals (CI) were determined from the reported crude estimates and population denominators, assuming exact binominal distribution as described by Clopper and Pearson. ${ }^{16}$ Heterogeneity between studies was assessed using the $\chi 2$-based Cochran's Q statistic and I-squared (I2) statistics ( $>50 \%$ representing moderate heterogeneity) and subgroup analysis was conducted to identify potential sources of heterogeneity. ${ }^{17}$ Publication bias assessment and meta-regression analysis were not conducted as the number of studies was less than ten for a given outcome. ${ }^{17} \mathrm{~A}$ 
p-value of $<0.05$ was considered statistically significant for the effect of study-level covariates on the estimated prevalence. All statistical analyses were conducted on Stata software version 16.1 (StataCorp LLC, College Station, Texas, USA) using the "metaprop" command. ${ }^{18}$

\section{Results}

\subsection{Search results-}

The PRISMA flowchart demonstrated the successive steps taken to select articles in the present systematic review (Fig. 1). We identified 216 publications through the PubMed database in total and one article was removed in initial screening because of duplication. Later on, 173 articles were removed after the screening of the title and abstract and by considering inclusion and exclusion criteria. After the screening process, 42 articles were assessed for eligibility, and further, 20 articles were excluded after the careful reading of their full text, and by considering the inclusion and exclusion criteria. Following the eligibility assessment, 22 articles were included in the systematic review.

\subsection{Study characteristics-}

Details and outcomes related to the mental health of included articles are provided in Table 1. ${ }^{3,19-39}$ All 22 cross-sectional studies were carried out in the Indian population, with age ranging from 12 to 82 years, and the majority of study participants were above 18 years. These 22 studies enrolled a total of 9947 participants, including 5542 (55.7\%) males and $4405(44.3 \%)$ females. Ten studies included measures of depression and stress while eight studies included measures of anxiety. Four studies assessed measures of sleep disturbances or abnormality, and two studies assessed psychological distress/stress while one study assessed overall mental health status. It was additionally observed that one study each used measures of suicidal ideas and substance or illegal drug use, quality of life (QoL), paranoia, and distress related to social media. Two studies did not explicitly report the overall prevalence rates of symptoms instead reported mean score and SD for psychological symptoms.

\subsection{Quality appraisal-}

In our review, we used "NIH quality assessment tools" for the assessment of the quality of data of the included studies. The result of the quality assessment of the included articles is provided in a supplementary file. The quality assessment indicated that five (22.7\%) studies were of poor quality and seven (31.8\%) studies were of good quality while the rest of included studies, 10 (45.5\%) were of fair quality.

\subsection{Measurement tools-}

Table 1 presents the scales used to assess the psychological wellbeing of the health care professionals and the general population. Various type of scales was used for assessing the prevalence of different adverse psychological outcomes in the included studies $(n=22)$. A generalized Anxiety Disorder (GAD-7) questionnaire was used for measuring anxiety symptoms in three studies. 9-item Patient Health Questionnaire (PHQ-9), and the Centre for Epidemiological Studies Depresion (CES-D) scale were used for measuring depressive symptoms in four and one studies respectively. The Perceived Stress Scale 10 (PSS10) was used for measuring stress symptoms in three studies. General Health Questionnaire-28 (GHQ-28) was used for measuring psychological distress symptoms in one study. The Hospital Anxiety and Depression (HADS) Scale was used for measuring anxiety and depression symptoms in one study. The Depression, Anxiety, and Stress Scale- 21 items (DASS-21) was used for the evaluation of depression, anxiety, and stress symptoms in three studies. Other authors included a validated selfreported questionnaire for the assessment of adverse psychological outcomes.

\subsection{Prevalence of psychological outcomes-}

Fig. 2 showed the pooled prevalence of different psychological wellbeing. The prevalence of stress in the Indian population during the COVID-19 pandemic was reported in nine studies and the pooled prevalence was $60.7 \%$ (95\% CI: $42.3 \%-77.7 \%)$. The prevalence of
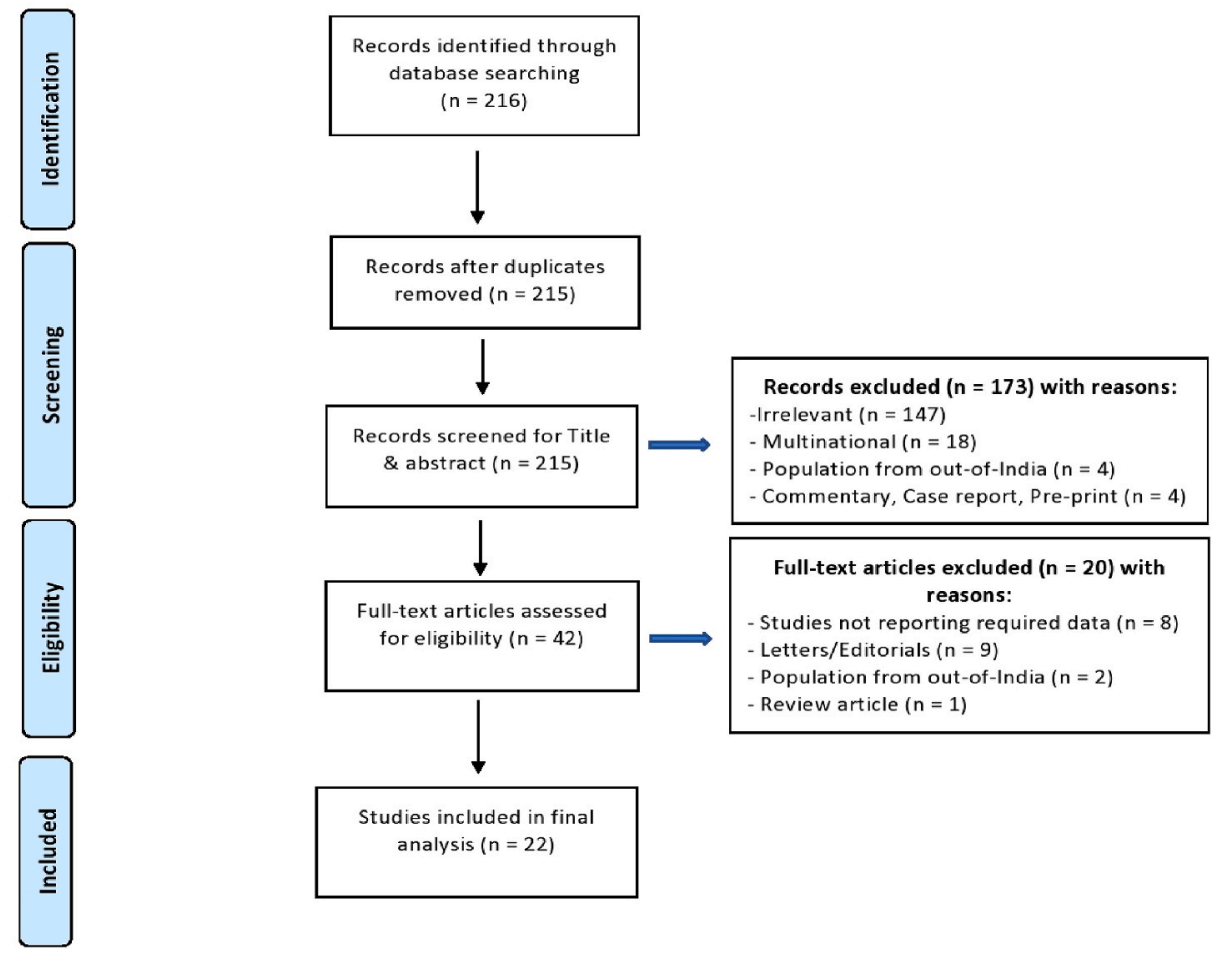

Fig. 1. Systematic search according to PRISMA guidelines. 
Table 1

Characteristics of included studies in this review.

\begin{tabular}{|c|c|c|c|c|c|c|c|c|c|c|}
\hline $\begin{array}{l}\text { Author, } \\
\text { Publication period }\end{array}$ & State/Area & $\begin{array}{l}\text { Study } \\
\text { design }\end{array}$ & $\begin{array}{l}\text { Total } \\
\text { participants }\end{array}$ & Target population & $\begin{array}{l}\text { Age (in } \\
\text { years) }\end{array}$ & $\begin{array}{l}\text { Male } \\
\text { gender } \\
(\%)\end{array}$ & $\begin{array}{l}\text { Data collection } \\
\text { method }\end{array}$ & Assessment tools & Cut-off & outcomes studied \\
\hline $\begin{array}{l}\text { Agarwal et al. }{ }^{19} \\
\text { September- } 2020\end{array}$ & $\begin{array}{l}\text { Uttar- } \\
\text { Pradesh }\end{array}$ & $\begin{array}{l}\text { Cross- } \\
\text { sectional }\end{array}$ & 89 & Type 1 diabetes (T1D) patients & $\begin{array}{l}\text { Mean age: } \\
(19.61 \pm \\
\text { 3.8), Range: } \\
12-24\end{array}$ & $\begin{array}{l}46 \\
(51.69 \%)\end{array}$ & Online survey & PSS-10 & $\begin{array}{l}\text { PSS-10 score (14 \& } \\
\text { above) }\end{array}$ & $\begin{array}{l}\text { Moderate stress and } \\
\text { severe stress }\end{array}$ \\
\hline $\begin{array}{l}\text { Chakraborty } \\
\text { et al. } \\
\text { May- } 2020\end{array}$ & West Bengal & $\begin{array}{l}\text { Cross- } \\
\text { sectional }\end{array}$ & 507 & General population & $\begin{array}{l}\text { Mean age: } \\
(33.9 \pm \\
8.27) \\
\text { Range: } 18- \\
67\end{array}$ & $\begin{array}{l}382 \\
(75.35 \%)\end{array}$ & Online survey & $\begin{array}{l}\text { 38-item self-designed } \\
\text { questionnaire }\end{array}$ & - & $\begin{array}{l}\text { Depression and } \\
\text { disturbed sleep-wake } \\
\text { cycle. }\end{array}$ \\
\hline $\begin{array}{l}\text { Chatterjee et al. } \\
\text { May- } 2020\end{array}$ & West Bengal & $\begin{array}{l}\text { Cross- } \\
\text { sectional }\end{array}$ & 152 & Doctors & $\begin{array}{l}\text { Mean age: } \\
(42.05 \pm \\
12.19)\end{array}$ & $\begin{array}{l}119 \\
(78.29 \%)\end{array}$ & Online survey & DASS-21 & - & $\begin{array}{l}\text { Depression, anxiety \& } \\
\text { stress }\end{array}$ \\
\hline $\begin{array}{l}\text { Dabholkar et al. }{ }^{22} \\
\text { June- } 2020\end{array}$ & Maharashtra & $\begin{array}{l}\text { Cross- } \\
\text { sectional }\end{array}$ & 40 & $\begin{array}{l}\text { COVID-19 infected health care } \\
\text { professionals (HCPs) }\end{array}$ & - & $16(40 \%)$ & $\begin{array}{l}\text { Both interview } \\
\text { and an online } \\
\text { questionnaire } \\
\text { form }\end{array}$ & $\begin{array}{l}\text { Self-reported } \\
\text { questionnaire }\end{array}$ & - & $\begin{array}{l}\text { Several psychological } \\
\text { parameters like } \\
\text { anxiety, fear, anger, } \\
\text { irritability and } \\
\text { insomnia. }\end{array}$ \\
\hline $\begin{array}{l}\text { Das et al. } \\
\text { October-2020 }\end{array}$ & Across India & $\begin{array}{l}\text { Cross- } \\
\text { sectional }\end{array}$ & 422 & Frontline doctors & $\begin{array}{l}\text { Mean age: } \\
(27.61 \pm \\
4.98)\end{array}$ & $\begin{array}{l}224 \\
(53.08 \%)\end{array}$ & online survey & PHQ-9, PSS-10 & $\begin{array}{l}\text { PHQ-9 score }>4 \\
\text { PSS-10 score }>13\end{array}$ & Depression \& stress \\
\hline $\begin{array}{l}\text { Ghosh et al. }{ }^{24} \\
\text { May- } 2020\end{array}$ & Delhi & $\begin{array}{l}\text { Cross- } \\
\text { sectional }\end{array}$ & 150 & Type 2 diabetes (T2DM) patients & - & $93(62 \%)$ & $\begin{array}{l}\text { Telephonic } \\
\text { interview }\end{array}$ & $\begin{array}{l}\text { Self-reported } \\
\text { questionnaire }\end{array}$ & - & $\begin{array}{l}\text { Mental stress and sleep } \\
\text { disturbances. }\end{array}$ \\
\hline $\begin{array}{l}\text { Gupta et al. }{ }^{3} \text { July- } \\
2020\end{array}$ & Across India & $\begin{array}{l}\text { Cross- } \\
\text { sectional }\end{array}$ & 1124 & Health care workers (HCWs) & - & $\begin{array}{l}718 \\
(63.88 \%)\end{array}$ & Online survey & HADS Scale & $\begin{array}{l}\text { HADS score } 8 \& \\
\text { above }\end{array}$ & Depression \& anxiety \\
\hline $\begin{array}{l}\text { Khanna et al }{ }^{25} \\
\text { June- } 2020\end{array}$ & Across India & $\begin{array}{l}\text { Cross- } \\
\text { sectional }\end{array}$ & 2355 & Ophthalmologists & $\begin{array}{l}\text { Mean age: } \\
(42.5 \pm \\
12.05), \\
\text { Range: } 25- \\
82\end{array}$ & $\begin{array}{l}1332 \\
(56.56 \%)\end{array}$ & Online survey & PHQ-9 & - & Depression \\
\hline $\begin{array}{l}\text { Mishra et al. }{ }^{26} \\
\text { June- } 2020\end{array}$ & Across India & $\begin{array}{l}\text { Cross- } \\
\text { sectional }\end{array}$ & 716 & Ophthalmology trainees & $\begin{array}{l}\text { Mean age: } \\
(29.1 \pm \\
3.14), \\
\text { Range: } 23- \\
45\end{array}$ & $\begin{array}{l}275 \\
(38.41 \%)\end{array}$ & Online survey & $\begin{array}{l}\text { self-reported } \\
\text { questionnaire }\end{array}$ & - & Stress \\
\hline $\begin{array}{l}\text { Muruganandam } \\
\text { et al. }^{27} \text { June- } \\
2020\end{array}$ & South India & $\begin{array}{l}\text { Cross- } \\
\text { sectional }\end{array}$ & 132 & $\begin{array}{l}\text { patients diagnosed with severe } \\
\text { mental illness [SMI] and their } \\
\text { primary caregivers }\end{array}$ & $\begin{array}{l}\text { Mean age: } \\
(33.9 \pm \\
10.9), \\
\text { Range: } 21- \\
52\end{array}$ & $\begin{array}{l}63 \\
(47.73 \%)\end{array}$ & Telephonic survey & 23-item questionnaire & - & $\begin{array}{l}\text { Impaired sleep, } \\
\text { suicidal ideas and } \\
\text { taking substances or } \\
\text { illegal drugs. }\end{array}$ \\
\hline $\begin{array}{l}\text { Pandey et al. }{ }^{28} \\
\text { September-2020 }\end{array}$ & $\begin{array}{l}\text { Uttar- } \\
\text { Pradesh }\end{array}$ & $\begin{array}{l}\text { Cross- } \\
\text { sectional }\end{array}$ & 83 & $\begin{array}{l}\text { Final year medical students and } \\
\text { Obstetrics and Gynaecology } \\
\text { trainees }\end{array}$ & $\begin{array}{l}80(96.4 \%) \\
\text { were aged } \\
<30 \text { years } \\
\text { old }\end{array}$ & $\begin{array}{l}36 \\
(43.37 \%)\end{array}$ & $\begin{array}{l}\text { Online } \\
\text { questionnaire } \\
\text { form }\end{array}$ & GAD-7 and PHQ-9 & - & Depression \& anxiety \\
\hline $\begin{array}{l}\text { Podder et al. } \\
\text { July- } 2020\end{array}$ & $\begin{array}{l}\text { Eastern } \\
\text { India }\end{array}$ & $\begin{array}{l}\text { Cross- } \\
\text { sectional }\end{array}$ & 384 & $\begin{array}{l}\text { Dermatologists and other } \\
\text { physicians }\end{array}$ & $\begin{array}{l}\text { Mean age: } \\
(31.9 \pm 8.5)\end{array}$ & $\begin{array}{l}235 \\
(61.20 \%)\end{array}$ & $\begin{array}{l}\text { Web-based survey } \\
\text { (online) }\end{array}$ & PSS-10 & PSS-10 score $\geq 14$ & Stress \\
\hline \multirow[t]{2}{*}{$\begin{array}{l}\text { Rehman et al }{ }^{30} \\
\text { June- } 2020\end{array}$} & Across India & $\begin{array}{l}\text { Cross- } \\
\text { sectional }\end{array}$ & 390 & $\begin{array}{l}139 \text { students, } 51 \text { teachers, } 31 \\
\text { researchers, } 34 \text { mental health } \\
\text { professionals, } 33 \text { health } \\
\text { professionals (Doctors and } \\
\text { Nurses), } 35 \text { in a corporate job, } 80 \\
\text { were others. }\end{array}$ & $\begin{array}{l}\text { Mean age: } \\
28.95\end{array}$ & $\begin{array}{l}108 \\
(27.69 \%)\end{array}$ & Online survey & DASS-21 & $\begin{array}{l}\text { Depression }>9 \text {, } \\
\text { Anxiety }>7 \text {, Stress } \\
>14\end{array}$ & $\begin{array}{l}\text { Depression, anxiety \& } \\
\text { stress }\end{array}$ \\
\hline & Across India & & 662 & General population & & & Online survey & & - & (continued on next page) \\
\hline
\end{tabular}




\begin{tabular}{|c|c|c|c|c|c|c|c|c|c|c|}
\hline $\begin{array}{l}\text { Author, } \\
\text { Publication period }\end{array}$ & State/Area & $\begin{array}{l}\text { Study } \\
\text { design }\end{array}$ & $\begin{array}{l}\text { Total } \\
\text { participants }\end{array}$ & Target population & $\begin{array}{l}\text { Age (in } \\
\text { years) }\end{array}$ & $\begin{array}{l}\text { Male } \\
\text { gender } \\
(\%)\end{array}$ & $\begin{array}{l}\text { Data collection } \\
\text { method }\end{array}$ & Assessment tools & Cut-off & outcomes studied \\
\hline $\begin{array}{l}\text { Roy et al. }{ }^{31} \\
\text { June- } 2020\end{array}$ & & $\begin{array}{l}\text { Cross- } \\
\text { sectional }\end{array}$ & & & $\begin{array}{l}\text { Mean age: } \\
(29.09 \pm \\
8.83)\end{array}$ & $\begin{array}{l}322 \\
(48.64 \%)\end{array}$ & & $\begin{array}{l}\text { Self-reported } \\
\text { questionnaire }\end{array}$ & & $\begin{array}{l}\text { Sleep difficulties, } \\
\text { paranoia and distress. }\end{array}$ \\
\hline $\begin{array}{l}\text { Sahu et al. } .^{32} \text { May- } \\
2020\end{array}$ & Across India & $\begin{array}{l}\text { Cross- } \\
\text { sectional }\end{array}$ & 611 & Orthopaedic surgeons & - & $\begin{array}{l}611 \\
(100 \%)\end{array}$ & $\begin{array}{l}\text { Online } \\
\text { anonymous } \\
\text { survey }\end{array}$ & $\begin{array}{l}\text { Validated questionnaire } \\
\text { using face validation and } \\
\text { content validation } \\
\text { techniques of Lawshe } \\
\text { criterion }\end{array}$ & - & Stress \\
\hline $\begin{array}{l}\text { Sharma AJ et al. }{ }^{33} \\
\text { September- } 2020\end{array}$ & Across India & $\begin{array}{l}\text { Cross- } \\
\text { sectional } \\
\text { study }\end{array}$ & 282 & Indian citizen & - & $\begin{array}{l}175 \\
(62.06 \%)\end{array}$ & Online survey & GAD-7 \& CESD-D scale & - & Depression \& anxiety \\
\hline $\begin{array}{l}\text { Sharma N et al. }{ }^{34} \\
\text { October-2020 }\end{array}$ & Across India & $\begin{array}{l}\text { Cross- } \\
\text { sectional }\end{array}$ & 537 & Women professionals & $\begin{array}{l}\text { Mean age: } \\
32.78\end{array}$ & - & $\begin{array}{l}\text { Online } \\
\text { questionnaire } \\
\text { form }\end{array}$ & 36-item questionnaire & - & Mental health affected \\
\hline $\begin{array}{l}\text { Suryakumari } \\
\text { et al. } \\
\text { September- } 2020\end{array}$ & Telangana & $\begin{array}{l}\text { Cross- } \\
\text { sectional }\end{array}$ & 307 & Dental Practitioners & $\begin{array}{l}\text { Age range: } \\
20-60\end{array}$ & $\begin{array}{l}145 \\
(47.23 \%)\end{array}$ & $\begin{array}{l}\text { Online } \\
\text { questionnaire } \\
\text { form }\end{array}$ & $\begin{array}{l}\text { A questionnaire by } \\
\text { Ahmed MA et al. }\end{array}$ & $\begin{array}{l}\text { Mean fear score was } \\
\text { calculated and taken } \\
\text { as a cut off value. }\end{array}$ & Fear and anxiety. \\
\hline $\begin{array}{l}\text { Suryavanshi } \\
\text { et al. } \\
\text { September- } 2020\end{array}$ & Across India & $\begin{array}{l}\text { Cross- } \\
\text { sectional }\end{array}$ & 197 & Healthcare professionals & - & $\begin{array}{l}96 \\
(48.73 \%)\end{array}$ & $\begin{array}{l}\text { Online } \\
\text { questionnaire } \\
\text { form }\end{array}$ & PHQ-9, GAD-7 & $\begin{array}{l}\text { Presence of any } \\
\text { depressive } \\
\text { symptoms PHQ-9 } \\
(\geq 5) \text {, Anxiety } \\
\text { symptoms GAD-7 } \\
(\geq 5) \text {. }\end{array}$ & $\begin{array}{l}\text { Depression, anxiety } \\
\text { and QoL. }\end{array}$ \\
\hline $\begin{array}{l}\text { Upadhyaya et al. }{ }^{37} \\
\text { July- } 2020\end{array}$ & Delhi & $\begin{array}{l}\text { Cross- } \\
\text { sectional }\end{array}$ & 138 & PG students in orthopaedics & - & $\begin{array}{l}138 \\
(100 \%)\end{array}$ & Online survey & $\begin{array}{l}\text { Self-reported } \\
\text { questionnaire }\end{array}$ & - & Stress \\
\hline $\begin{array}{l}\text { Venugopal et al al } \\
\text { August- } 2020\end{array}$ & Across India & $\begin{array}{l}\text { Cross- } \\
\text { sectional }\end{array}$ & 453 & General population & $\begin{array}{l}\text { Mean age: } \\
36.52\end{array}$ & $\begin{array}{l}225 \\
(49.67 \%)\end{array}$ & Online survey & GHQ-28 & $\begin{array}{l}\text { GHQ cut-off of } 23 \\
\text { ( } \leq 23: \text { without a } \\
\text { mental disorder) }\end{array}$ & Psychological distress \\
\hline $\begin{array}{l}\text { Verma et al. }{ }^{39} \\
\text { June- } 2020\end{array}$ & Across India & $\begin{array}{l}\text { Cross- } \\
\text { sectional }\end{array}$ & 354 & General population & - & $\begin{array}{l}183 \\
(51.70 \%)\end{array}$ & Online survey & DASS-21 & $\begin{array}{l}>14 \text { for depression, } \\
>10 \text { for anxiety and } \\
>19 \text { for stress. }\end{array}$ & $\begin{array}{l}\text { Depression, anxiety \& } \\
\text { stress }\end{array}$ \\
\hline
\end{tabular}

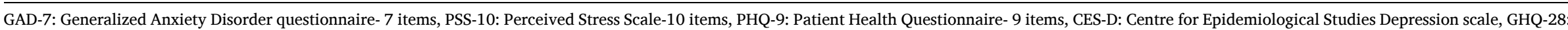
General Health Questionnaire-28 items, HADS: Hospital Anxiety and Depression Scale, DASS-21: Depression, Anxiety, and Stress Scale- 21 items. 


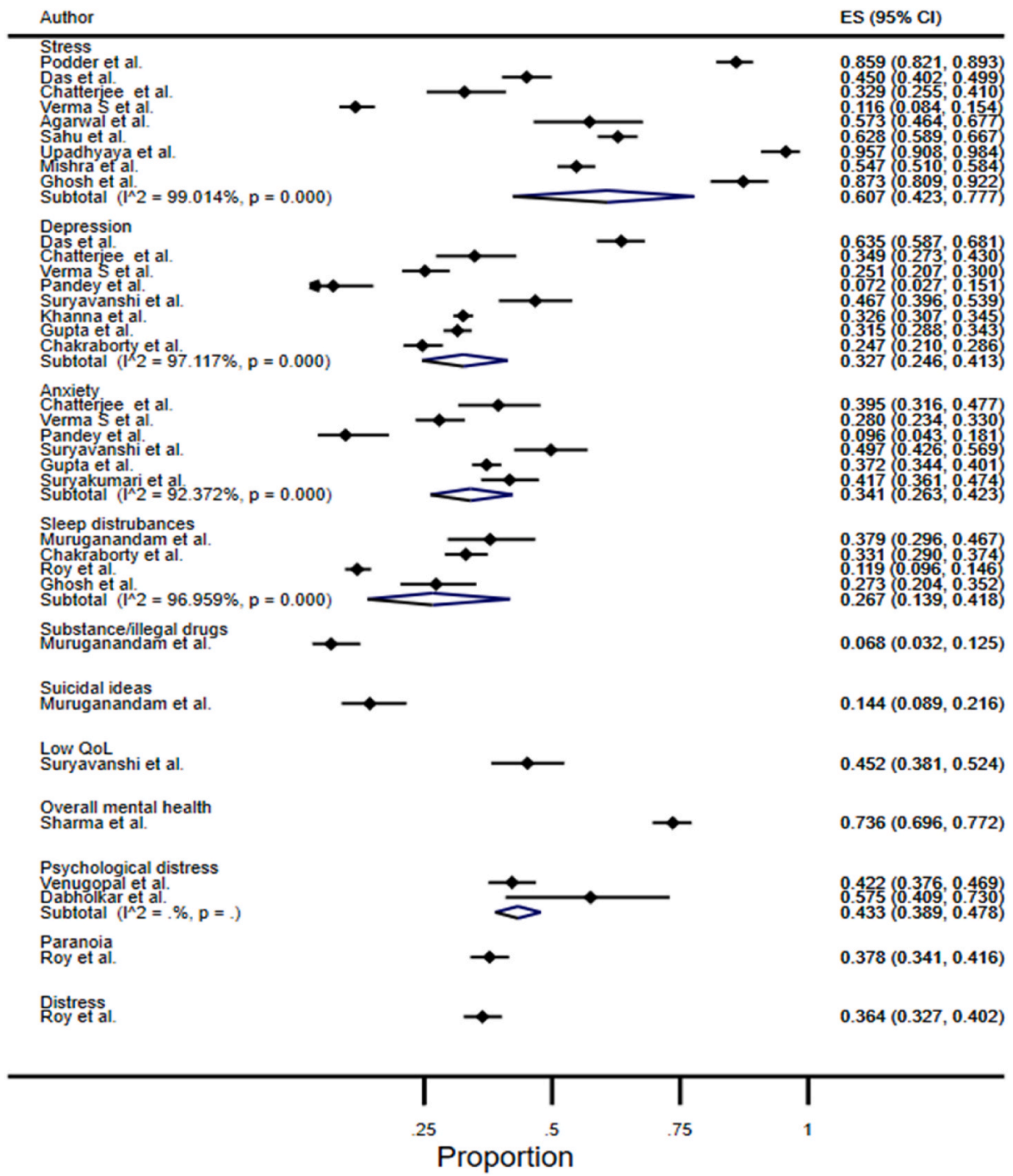

Fig. 2. The pooled prevalence of different psychological wellbeing.

depression was reported in eight studies and the pooled prevalence was $32.7 \%$ (95\% CI: $24.6 \%-41.3 \%$ ). The prevalence of anxiety was reported in six studies and the pooled prevalence was $34.1 \%$ (95\% CI: $26.3 \%-$ $42.3 \%)$. The prevalence of sleep disturbances or difficulty was reported in four studies and the pooled prevalence was $26.7 \%$ (95\% CI: $13.9 \%-$ $41.8 \%)$. The prevalence of psychological distress/stress was reported in two studies and the pooled prevalence was $43.3 \%$ (95\% CI: $38.9 \%-$ $47.8 \%)$. Prevalence of other outcomes such as suicidal ideas $(14.4 \%$, 95\% CI: 8.9\%-21.6\%) and use of substance/illegal drugs (6.8\%, 95\% CI: $3.2 \%-12.5 \%)$; low quality of life (45.2\%, $95 \%$ CI: $38.1 \%-52.4 \%)$; and impaired overall mental health (73.6\%, 95\% CI: 69.6\%-77.2\%) were also reported in single reports. As expected, high heterogeneity was observed in the above-mentioned outcomes (stress $\left(\mathrm{I}^{2}: 99.0 \%\right)$, anxiety $\left(\mathrm{I}^{2}: 97.1 \%\right)$, depression $\left(\mathrm{I}^{2}: 93.3 \%\right)$, and sleep disturbances $\left.\left(\mathrm{I}^{2}: 96.9 \%\right)\right)$.

On the other hand, two studies reported stress, anxiety, and depression in mean scores by gender as presented in Table 2 . We did not meta-analyzed these outcomes as different measurement scales were used, and none of the outcomes were statistically different between gender except for depression $(\mathrm{p}=0.041)$ in Sharma et al. indicating females had a higher mean score.

Table 2

Studies that presented stress, anxiety, and depression as mean scores by gender.

\begin{tabular}{|c|c|c|c|c|c|c|c|}
\hline \multirow[t]{2}{*}{ Author } & \multirow[t]{2}{*}{ Outcome } & \multirow[t]{2}{*}{ Sample size } & \multirow[t]{2}{*}{ Scale used } & \multicolumn{2}{|c|}{ Gender [mean (SD)] } & \multirow[t]{2}{*}{ Combined mean (SD) } & \multirow[t]{2}{*}{ P-value for gender within study } \\
\hline & & & & Male & Female & & \\
\hline \multirow[t]{2}{*}{ Sharma AJ et al. ${ }^{33}$} & & & & $(\mathrm{n}=175)$ & $(\mathrm{n}=107)$ & & \\
\hline & Anxiety & 282 & GAD-7 and CES-D & $6.15(5.82)$ & $6.90(5.25)$ & $6.44(5.61)$ & 0.277 \\
\hline \multirow{3}{*}{ Rehman et al. ${ }^{30}$} & Stress & 390 & DASS-21 & $12.65(9.26)$ & $13.44(9.72)$ & $13.22(9.60)$ & 0.467 \\
\hline & Anxiety & 390 & & $9.91(8.78)$ & $10.57(8.77)$ & $10.39(9.10)$ & 0.507 \\
\hline & Depression & 390 & & $10.81(9.99)$ & $10.96(9.76)$ & $10.92(9.82)$ & 0.893 \\
\hline
\end{tabular}

SD: standard deviation, GAD-7: Generalized Anxiety Disorder questionnaire- 7 items, CES-D: Centre for Epidemiological Studies Depression scale, DASS-21: Depression, Anxiety, and Stress Scale- 21 item. 


\subsection{Prevalence of psychological outcomes in sub-groups-}

Fig. 3 (a.) and (b.) showed the pooled prevalence of psychological wellbeing among health care workers (HCWs) and the general population, respectively.

The prevalence of stress was reported in six studies among HCWs and the pooled prevalence was $65.1 \%$ (95\% CI: $48.2 \%-80.3 \%$ ) while in three studies among the general population and the pooled prevalence was $51.7 \%$ (95\% CI: $05.3 \%-96.2 \%$ ). The prevalence of depression was reported in six studies among HCWs and the pooled prevalence was $35.4 \%$ (95\% CI: $25.1 \%-46.4 \%$ ) while in two studies among the general population and the pooled prevalence was $24.9 \%$ (95\% CI: $22.0 \%-27.8 \%$ ). The prevalence of anxiety was reported in five studies among HCWs and the pooled prevalence was $35.3 \%$ (95\% CI: $26.3 \%-44.9 \%$ ) while in one study among the general population and the prevalence was $28.0 \%$ (95\% CI: $23.4 \%-33.0 \%$ ). The prevalence of psychological distress reported among HCWs in one study was $57.5 \%$ (95\% CI: 40.9\%-73.0\%), while in the general population in one study was $42.2 \%$ (95\% CI: $37.6 \%-$ $46.9 \%$ ). The prevalence of sleep disturbances or difficulty was reported in four studies among the general population and the pooled prevalence was $26.7 \%$ (95\% CI: $13.9 \%-41.8 \%$ ).

Prevalence of other outcomes such as low quality of life $(45.2 \%, 95 \%$ CI: $38.1 \%-52.4 \%)$ and impaired overall mental health $(73.6 \%$, 95\% CI: 69.6\%-77.2\%) were also reported in single reports among HCWs while substance/illegal drugs (6.8\%, 95\% CI: $3.2 \%-12.5 \%)$ \& suicidal ideas (14.4\%, 95\% CI: 8.9\%-21.6\%); and paranoia (37.8\%, 95\% CI: $34.1 \%-$ $41.6 \%$ ) were also reported in single reports among general population.

\section{Discussion}

The sudden emergence of previously unknown disease (later named COVID-19 by WHO), followed by its rapid widespread transmission and declaration of a pandemic, has aggravated anxiety and stress levels in populations globally, leading to mental health problems in individuals. In the present systematic review and meta-analysis, we found that the COVID-19 pandemic has a significant impact on mental health outcomes. The articles used in our meta-analysis were cross-sectional studies. Variations in prevalence rates across studies were noticed, which could have resulted from different measurement scales with their different scores. For example, some studies reported any participants with scores above the cut-off point, while others only included participants with moderate-to-severe symptoms. According to our metaanalysis, the prevalence of stress, anxiety, depression, sleep

(a) Health care workers

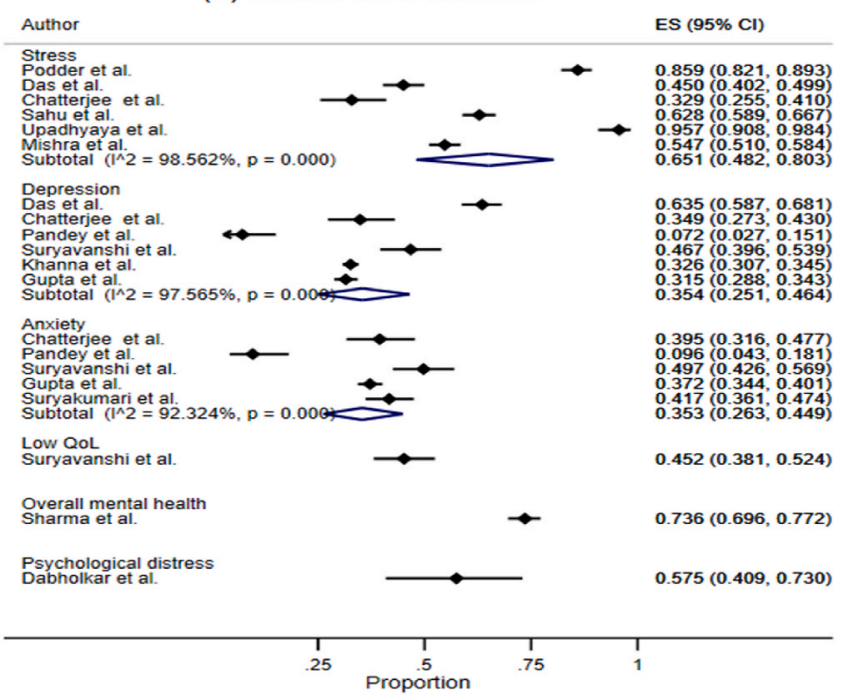

disturbances, and psychological distress because of the COVID-19 pandemic in Indians are $60.7 \%, 34.1 \%, 32.7 \%, 26.7 \%$, and $43.3 \%$ respectively. Sub-group analysis shows that Health Care Workers (HCWs) have a higher prevalence of stress, anxiety, depression \& psychological distress in comparison to the general population in the Indian context. In our meta-analysis sleep, disturbances were reported among the general population (26.7\%) only, but not for HCPs.

A meta-analysis including studies from 10 countries across the globe reported that pooled prevalence of stress $(29.6 \%$, 95\% CI: $24.3 \%-$ $35.4 \%)$, anxiety $(31.9 \%, 95 \%$ CI: $27.5 \%-36.7 \%)$, and depression (33.7\%, 95\% CI: $27.5 \%-40.6 \%$ ) among the general population during the COVID-19 pandemic. ${ }^{40} \mathrm{GOI}$ imposed many restrictions for curtailing the spread of COVID-19 such as travel restrictions, complete nationwide lockdown, isolation of cases, and quarantine of exposed persons in dedicated facilities. All these led to the making of huge changes to the daily routines of the people. It is a challenge to get accustomed to new ways of living such as working from home, temporary loss of income, online schooling of children, and lack of physical contact with other family members, friends and colleagues, and others. Adapting to such lifestyle changes and concerns related to a disease such as fear of contracting the disease, spreading to near and dear ones, and protecting the vulnerable persons in the family is a challenging and stressful task for most people. The current COVID-19 outbreak has triggered social stigma and discriminatory behaviors against people of certain cultural backgrounds and those who have been in contact with the virus. ${ }^{41}$ Stigma carries serious consequences including stimulating fear, anger, and intolerance directed at other people. People exposed to stigma are more likely to experience worse psychological well-being. The public health response to COVID-19 such as social distancing norms, travel bans, movement, restrictions, quarantines, etc. in itself carries the risk of increasing stigma and causing discrimination. ${ }^{42}$ All such circumstances are liable to affect the mental health of people in general and can be more difficult for people with pre-existing mental health problems. Some of the measures recommended for improving the mental health of the general population are: promoting healthy behaviors, maintaining social contacts with family and friends digitally, relying on information from authentic sources, creating awareness regarding recognizing mental problems early and seeking timely care, etc.

In the times before the COVID-19 pandemic, mental health issues have been recognized among health care professionals and several studies have demonstrated a higher burden of depression, anxiety, substance abuse, suicide, etc among health care workers. A recent metaanalysis including studies conducted in countries from the different (b) General population

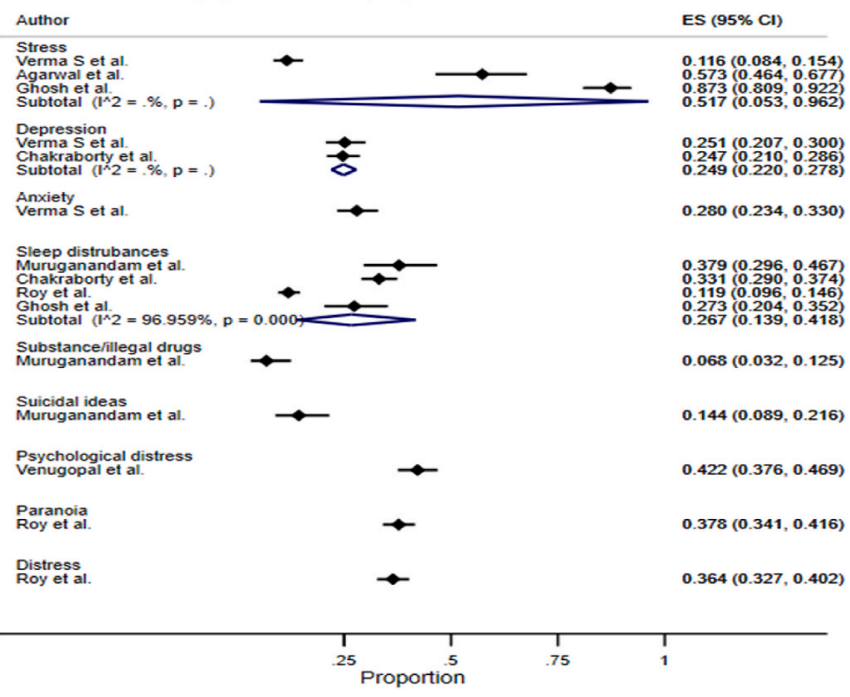

Fig. 3. (a.) and (b.): The pooled prevalence of psychological wellbeing among health care workers (HCWs) and general population, respectively. 
continents of the world reported that pooled prevalence of general health concerns $(62.5 \%, 95 \%$ CI: $57.0 \%-67.8 \%)$, psychological distress (37.8\%, 95\% CI: $28.4 \%-48.2 \%)$, anxiety features $(29.0 \%$, 95\% CI: $14.2 \%-50.3 \%)$ and depressive symptoms $(26.3 \%$, 95\% CI: $12.5 \%-$ 47.1\%) among health care workers (HCWs) exposed to SARS/MERS/ COVID-19. ${ }^{43}$ In our meta-analysis the prevalence of mental health problems were higher among HCWs as compared to reported prevalence in studies from other countries. This could be due to the current pandemic with India having the second-highest number of cases in the world posing a huge challenge on the health care staff who were unprepared. Health care workers are at a higher risk of psychological problems due to multiple factors. These include social isolation; stigma and social discrimination at workplaces and surroundings; higher risk of contracting the disease as they are exposed to patients with high viral load; fear of transmitting the disease to family members; excessive workload with long working hours; inadequate personal protective equipment. ${ }^{44}$ The health care professionals are faced with the challenge of assimilating a large amount of information in a short period and acquire new technical skills for properly handling the pandemic. Health authorities must address the concerns of health care professionals and should support them during such major health crises. Some measures recommended are providing adequate infrastructure, recruiting sufficient human resources to support the overburdened staff, ensuring the availability of adequate amounts of personal protective equipment (PPE), training of health care staff with regards to diagnosis, management, and prevention of COVID-19, etc.

The major strength of the present study is that we have tried to provide a comprehensive review on the overall burden of mental health problems during the COVID-19 pandemic in India with estimates for the general population and health care workers. This will help policymakers and health authorities to prioritize and address this important public health aspect while focusing on their efforts to control the pandemic situation.

Future research studies should be conducted to identify sociocultural and behavioural risk factors of adverse mental health outcomes so that effective prevention strategies can be planned. It is also suggested that future epidemiological studies using cohort study designs should be considered so that the changes in mental health outcomes can be evaluated over time.

Our study has several limitations. First, we searched a single database Medline (via PubMed) for the study selection process and might have missed a small number of pertinent research articles. Second, all included studies were cross-sectional \& prone to bias. Third, high heterogeneity was observed in evaluated outcomes. Fourth, although we did an extensive search, we may have inadvertently missed relevant studies. Fifth, the Majority of studies collected data in online mode by distributing the study questionnaires using different digital platforms, this could result in bias due to exclusion of potential participants. Finally, because of challenges in conducting a research on account of various restrictions during a pandemic, several studies were of suboptimal design.

\section{Conclusion \& recommendation}

A significant impact on psychological well-being during COVID-19 was observed in India as common adverse outcomes were stress (61\%), psychological distress (43\%), anxiety (34\%), depression (33\%), and sleep disturbances (27\%). Health Care Workers had a higher prevalence of stress, anxiety, depression \& psychological distress in comparison to the general population. Thus COVID-19 pandemic represents an unprecedented threat to mental health. HCWs need to be given priority to the prevention of mental health problems as they are the frontline workers in the current pandemic. As the pandemic leads to greater demand on the limited mental health care resources, the government policy should address the mental health problems in times of pandemics and should formulate strategies to improve the mental health in the general population.

\section{Appendix A. Supplementary data}

Supplementary data to this article can be found online at https://doi. org/10.1016/j.cegh.2021.100737.

\section{References}

1 Eurosurveillance Editorial Team. Note from the editors: World Health Organization declares novel coronavirus (2019-nCoV) sixth public health emergency of international concern. Euro Surveill [Internet]. 2020;25(5):200131e. https://doi.org/ 10.2807/1560-7917.ES.2020.25.5.200131e.

2 Cucinotta D, Vanelli M. WHO declares COVID-19 a pandemic. Acta Biomed. 2020;91 (1):157-160.

3 Gupta S, Prasad AS, Dixit PK, Padmakumari P, Gupta S, Abhisheka K. Survey of prevalence of anxiety and depressive symptoms among 1124 healthcare workers during the coronavirus disease 2019 pandemic across India. Med J Armed Forces India [Internet]. 2020:1-9. https://doi.org/10.1016/j.mjafi.2020.07.00.

4 Asmundson GJ, Taylor S. How health anxiety influences responses to viral outbreaks like COVID-19: what all decision-makers, health authorities, and health care professionals need to know. J Anxiety Disord. 2020;71:102211e.

5 Pakenham KI, Landi G, Boccolini G, Furlani A, Grandi S, Tossani E. The moderating roles of psychological flexibility and inflexibility on the mental health impacts of COVID-19 pandemic and lockdown in Italy. J Context Behav Sci J. 2020;17:109-118.

6 Hooper MW, Nápoles AM, Pérez-Stable EJ. COVID-19 and racial/ethnic disparities. JAMA [Internet]. 2020;323(24):2466-2467. https://doi.org/10.1001/ jama.2020.8598.

7 Roca M, Gili M. Economic crisis and mental health in Spain. Lancet. 2013;382(9909), 1977-8.

8 Moreno C, Wykes T, Galderisi S, et al. How mental health care should change as a consequence of the COVID-19 pandemic. Lancet Psychiatry [Internet. 2020;7 (September):813-824. https://doi.org/10.1016/S2215-0366(20)30307-2.

9 Roy A, Singh AK, Mishra S, Chinnadurai A, Mitra A, Bakshi O. Mental health implications of COVID-19 pandemic and its response in India. Int J Soc Psychiatr. 2020;1-14.

10 Ghebreyes TA. Addressing mental health needs: an integral part of COVID-19 response. World Psychiatr. 2020;19(2):129-130.

11 United Nations. Policy Brief : COVID-19 and the need for action on mental health. EXECUTIVE SUMMARY [Internet]; 2020:1-17. https://www.un.org/sites/un2.un. org/files/un_policy_brief-covid_and_mental_health_final.pdf.

12 WHO. Coronavirus Disease (COVID-19) Dashboard | [Internet]. WHO; 2020 [cited 2020 Nov 10] https://covid19.who.int/.

13 Moher D, Liberati A, Tetzlaff J, et al. Preferred reporting items for systematic reviews and meta-analyses: the PRISMA statement. Ann Intern Med. 2009;151(4):264-269.

14 https://www.nhlbi.nih.gov/health-topics/study-qualityassessment-tools.

15 DerSimonian R, Laird N. Meta-analysis in clinical trials. Contr Clin Trials. 1986;7(3): $177-188$.

16 Clopper CJ, Pearson ES. The use of confidence or fiducial limits illustrated in the case of the binomial. Biometrika. 1934;26(4):404-413.

17 Higgins JPT, Thomas J, Chandler J, et al., eds. Cochrane Handbook for Systematic Reviews of Interventions. second ed. Chichester (UK): John Wiley \& Sons; 2019.

18 Nyaga VN, Arbyn M, Aerts M. Metaprop: a Stata command to perform meta-analysis of binomial data. Arch Publ Health. 2014;72:39. https://doi.org/10.1186/2049-325872-39.

19 Agarwal N, Harikar M, Shukla R, Bajpai A. COVID-19 pandemic: a double trouble for Indian adolescents and young adults living with type 1 diabetes. Int J Diabetes Dev Ctries. 2020;40(3):346-352.

20 Chakraborty K, Chatterjee M. Psychological impact of COVID-19 pandemic on general population in West Bengal : a cross-sectional study. Indian J Psychiatr. 2020; 62(3):266-272.

21 Chatterjee SS, Bhattacharyya R, Bhattacharyya S, Gupta S, Das S, Banerjee BB. Attitude, practice, behavior, and mental health impact of COVID-19 on doctors. Indian J Psychiatr. 2020;62(3):257-265.

22 Dabholkar YG, Sagane BA, Dabholkar TY, Divity S. COVID19 infection in health care Professionals : risks, work- safety and psychological issues. Indian J Otolaryngol Head Neck Surg. 2020;72(4):468-473. https://doi.org/10.1007/s12070-020-01928-4.

23 Das A, Sil A, Jaiswal S, et al. A study to evaluate depression and perceived stress among frontline Indian doctors combating the COVID-19 pandemic. Prim Care Companion CNS Disord. 2020;22(5):20m02716.

24 Ghosh A, Arora B, Gupta R, Anoop S, Misra A. Effects of nationwide lockdown during COVID-19 epidemic on lifestyle and other medical issues of patients with type 2 diabetes in north India. Diabetes Metab Syndr Clin Res Rev. 2020;14:917-920.

25 Khanna RC, Honavar SG, Metla AL, Bhattacharyya A, Maulik PK. Psychological impact of COVID-19 on ophthalmologists-in-training and practising ophthalmologists in India. Indian J Ophthalmol. 2020;68(6):994-998. http://www. ncbi.nlm.nih.gov/pubmed/28331284\%0Ahttp://www.pubmedcentral.nih.gov/arti clerender.fcgi?artid=PMC5354527\%5Cnhttp://bmcpsychiatry.biomedcentral.co m/articles/10.1186/1471-244X-11-49\%5Cnhttp://bmcophthalmol.biomedcentral. com/articles/10.1186/s12886.

26 Mishra D, Nair AG, Gandhi RA, et al. The impact of COVID-19 related lockdown on ophthalmology training programs in India - outcomes of a survey. Indian $J$ Ophthalmol. 2020;68(6):999-1004. http://www.ncbi.nlm.nih.gov/pubmed 
/28331284\%0Ahttp://www.pubmedcentral.nih.gov/articlerender.fcgi?artid=PMC5 354527\%5Cnhttp://bmcpsychiatry.biomedcentral.com/articles/10.

1186/1471-244X-11-49\%5Cnhttp://bmcophthalmol.biomedcentral.com/arti cles/10.1186/s12886.

27 Muruganandam P, Neelamegam S, Menon V, Alexander J. COVID-19 and Severe Mental Illness: impact on patients and its relation with their awareness about COVID19. Psychiatr Res. 2020;291:113265.

28 Pandey U, Corbett G, Mohan S, et al. Anxiety, depression and behavioural changes in junior doctors and medical students associated with the coronavirus pandemic: a cross-sectional survey. J Obstet Gynaecol India. 2020;1-5. https://doi.org/10.1007/ s13224-020-01366-w.

29 Podder I, Agarwal K, Datta S. Comparative analysis of perceived stress in dermatologists and other physicians during national lock-down and COVID-19 pandemic with exploration of possible risk factors: a web-based cross-sectional study from Eastern India. Dermatol Ther. 2020;33(4). e13789.

30 Rehman U, Shahnawaz MG, Khan NH, et al. Depression, anxiety and stress among Indians in times of covid-19 lockdown. Community Ment Health J. 2020:1-7. https:// doi.org/10.1007/s10597-020-00664-x.

31 Roy D, Tripathy S, Kar SK, Sharma N, Verma SK, Kaushal V. Study of knowledge, attitude, anxiety \& perceived mental healthcare need in Indian population during COVID-19 pandemic. Asian J Psychiatr. 2020;51:102083. https://doi.org/10.1016/j. ajp.2020.102083.

32 Sahu D, Agrawal T, Rathod V, Bagaria V. Impact of COVID 19 lockdown on orthopaedic surgeons in India: a survey. J Clin Orthop Trauma. 2020;11:S283-S290. https://doi.org/10.1016/j.jcot.2020.05.007.

33 Sharma AJ, Subramanyam MA. A cross-sectional study of psychological wellbeing of Indian adults during the Covid-19 lockdown : different strokes for different folks. PloS One. 2020;15(9). https://doi.org/10.1371/journal.pone.0238761. e0238761.

34 Sharma N, Vaish H. Impact of COVID - 19 on mental health and physical load on women professionals : an online cross-sectional survey. Health Care Women Int. 2020; Oct 6:1-18. https://doi.org/10.1080/07399332.2020.1825441.
35 Suryakumari VBP, Pallavi Reddy Y, Yadav SS, Doshi D, Surekha Reddy V. Assessing fear and anxiety of corona virus among dental practitioners. Disaster Med Public Health Prep. 2020 Sep 11:1-6. https://doi.org/10.1017/dmp.2020.350. Epub ahead of print. PMID: 32921326.

36 Suryavanshi N, Kadam A, Dhumal G, et al. Mental health and quality of life among healthcare professionals during the COVID-19 pandemic in India. Brain Behav. 2020. https://doi.org/10.1002/brb3.1837, 00:e01837.

37 Upadhyaya GK, Jain VK, Iyengar KP, Patralekh MK, Vaish A. Impact of COVID-19 on post-graduate orthopaedic training in Delhi-NCR. J Clin Orthop Trauma. 2020;11: S687-S695. https://doi.org/10.1016/j.jcot.2020.07.018.

38 Venugopal VC, Mohan A, Chennabasappa LK. Status of mental health and its associated factors among the general populace of India during COVID-19 pandemic. Asia Pac Psychiatr. 2020. https://doi.org/10.1111/appy.12412. e12412.

39 Verma S, Mishra A. Depression, anxiety, and stress and socio-demographic correlates among general Indian public during COVID-19. Int J Soc Psychiatr. 2020;66(8): 756-762.

40 Salari N, Hosseinian-Far A, Jalali R, et al. Prevalence of stress, anxiety, depression among the general population during the COVID-19 pandemic: a systematic review and meta-analysis. Glob Health. 2020:1-11.

41 Selvaraj S, Reddy PK, Murlidharan K. Impact of COVID-19 on mental health: a watershed moment in tertiary care service provision in India? Asian J Psychiatr. 2020;54:102229.

42 Khanagar SB, Al-Ehaideb A, Vishwanathaiah S, Maganur PC, Varadarajan S, Patil S. Depression, anxiety, and psychological distress among health-care providers during the outbreak of the life-threatening coronavirus disease (COVID-19). J Contemp Dent Pract. 2020;21(5):471-472.

43 Pablo GS De, Vaquerizo-serrano J, Catalan A, et al. Impact of coronavirus syndromes on physical and mental health of health care workers: systematic review and metaanalysis. J Affect Disord. 2020;275:48-57.

44 Nanjundaswamy MH, Pathak H, Chaturvedi SK. Perceived stress and anxiety during COVID-19 among psychiatry trainees. Asian J Psychiatr. 2020;54:102282. 